\title{
Replication analysis identifies TYK2 as a multiple sclerosis susceptibility factor
}

\author{
Maria Ban*,1, An Goris ${ }^{2}$, Åslaug R Lorentzen ${ }^{3,4}$, Amie Baker ${ }^{1}$, Tania Mihalova ${ }^{5}$, \\ Gillian Ingram ${ }^{6}$, David R Booth ${ }^{7}$, Robert N Heard ${ }^{7}$, Graeme J Stewart ${ }^{7}$, Elke Bogaert ${ }^{2}$, \\ Bénédicte Dubois ${ }^{2}$, Hanne F Harbo ${ }^{3}$, Elisabeth G Celius ${ }^{3}$, Anne Spurkland ${ }^{8}$, \\ Richard Strange $^{5}$, Clive Hawkins ${ }^{5}$, Neil P Robertson ${ }^{6}$, Frank Dudbridge ${ }^{9}$, James Wason ${ }^{9}$, \\ Philip L De Jager ${ }^{10,11}$, David Hafler ${ }^{11}$, John D Rioux ${ }^{12}$, Adrian J Ivinson ${ }^{13}$, \\ Jacob L McCauley ${ }^{14}$, Margaret Pericak-Vance ${ }^{14}$, Jorge R Oksenberg ${ }^{15}$, Stephen L Hauser ${ }^{15}$, \\ David Sexton $^{16}$, Jonathan Haines ${ }^{16}$ and Stephen Sawcer ${ }^{1}$, The Wellcome Trust \\ Case-Control Consortium (WTCCC) and Alastair Compston ${ }^{1}$
}

\begin{abstract}
${ }^{1}$ Department of Clinical Neuroscience, Addenbrooke's, Hospital, University of Cambridge, Cambridge, UK; ${ }^{2}$ Section for Experimental Neurology, Katholieke Universiteit Leuven, Leuven, Belgium; ${ }^{3}$ Department of Neurology, Faculty Division Ullevål University Hospital, University of Oslo, Oslo, Norway; ${ }^{4}$ Institute of Immunology, Rikshospitalet University Hospital, Oslo, Norway; ${ }^{5}$ Department of Neurology, Keele University Medical School, Hartshill Campus, Stoke on Trent, UK; ${ }^{6}$ Department of Neurology, University Hospital of Wales, Heath Park, Cardiff, UK; ${ }^{7}$ Institute for Immunology and Allergy Research, Westmead Millennium Institute, Westmead Hospital, University of Sydney, New South Wales, Australia; ${ }^{8}$ Institute of Basic Medical Sciences, University of Oslo, Oslo, Norway; ${ }^{9}$ MRC Biostatistics Unit, Cambridge, UK; ${ }^{10}$ Harvard Medical School/Partners Healthcare Center for Genetics and Genomics, Boston, MA, USA; ${ }^{11}$ Division of Molecular Immunology, Center for Neurologic Diseases, Brigham and Women's Hospital, Boston, MA, USA; ${ }^{12}$ Broad Institute of Harvard University and Massachusetts Institute of Technology, Cambridge, MA, USA; ${ }^{13}$ Harvard NeuroDiscovery Center, Harvard Medical School, Boston, MA, USA; ${ }^{14}$ University of Miami School of Medicine, Miami, FL, USA; ${ }^{15}$ Department of Neurology, University of California San Francisco, San Francisco, CA, USA; ${ }^{16}$ Center for Human Genetics Research, Vanderbilt University Medical Center, Nashville, TN, USA
\end{abstract}

In a recent genome-wide association study (GWAS) based on 12374 non-synonymous single nucleotide polymorphisms we identified a number of candidate multiple sclerosis susceptibility genes. Here, we describe the extended analysis of 17 of these loci undertaken using an additional 4234 patients, 2983 controls and 2053 trio families. In the final analysis combining all available data, we found that evidence for association was substantially increased for one of the 17 loci, rs34536443 from the tyrosine kinase 2 (TYK2) gene $\left(P=2.7 \times 10^{-6}\right.$, odds ratio $=1.32(1.17-1.47))$. This single nucleotide polymorphism results in an amino acid substitution (proline to alanine) in the kinase domain of TYK2, which is predicted to influence the levels of phosphorylation and therefore activity of the protein and so is likely to have a functional role in multiple sclerosis.

European Journal of Human Genetics (2009) 17, 1309-1313; doi:10.1038/ejhg.2009.41; published online 18 March 2009

Keywords: multiple sclerosis; TYK2

*Correspondence: Dr M Ban, Department of Clinical Neurosciences, Addenbrooke's Hospital, Cambridge University, Box 165, Hills Road, Cambridge CB2 2QQ, UK.

Tel: + 441223 217222; Fax: + 441223 336943;

E-mail: mb531@medschl.cam.ac.uk

Received 28 August 2008; revised 4 February 2009; accepted 17 February 2009; published online 18 March 2009

\section{Introduction}

Multiple sclerosis is a disease of the central nervous system that is frequently disabling. ${ }^{1}$ Although evidence indicates that the condition results from a complex interplay 
between environmental and genetic factors, ${ }^{2-5}$ relatively little is known about precisely which genes are involved. Association with the DR15 haplotype from the major histocompatibility complex (MHC) on chromosome $6 \mathrm{p} 21$ has been established for more than 30 years $^{6}$ and confirmed in nearly every population studied. ${ }^{7}$ Identification of other susceptibility genes has proven to be decidedly more difficult. Systematic efforts to screen for linkage ${ }^{8}$ together with segregation analysis ${ }^{9,10}$ indicate that susceptibility to multiple sclerosis is likely determined by a series of common variants each exerting only a modest effect on risk. Fortunately, the resources necessary to find such modest genetic effects are now available and the last 2 years have seen remarkable progress in the identification of susceptibility genes of relevance in multiple sclerosis. Through a candidate gene approach prompted by a careful systematic analysis of all available data, association with the interleukin-7 receptor (IL7R) gene has been firmly established. ${ }^{11,12}$ In a genome-wide association study (GWAS) involving 931 cases, The International Multiple Sclerosis Genetics Consortium (IMSGC) identified the interleukin-2 receptor (IL2R) as a susceptibility gene, ${ }^{13}$ which was confirmed in a very large replication study. ${ }^{14}$ Recent studies have also shown a role for CD226 and CLEC16A in multiple sclerosis susceptibility. ${ }^{15-17}$

In parallel with these efforts, we completed a GWAS based on 12374 non-synonymous single nucleotide polymorphisms (nsSNPs) typed in 975 patients and 1466 controls. This GWAS was performed as part of The Wellcome Trust Case-Control Consortium (WTCCC) ${ }^{18}$ (A full list of WTCCC contributors is provided in the Supplementary information online). Although this study failed to identify any unequivocally associated loci, other than those in linkage disequilibrium (LD) with DR15, the most highly ranked markers implicated a number of promising candidate genes. Through extending the analysis of the most promising 17 of these loci, we have now identified another non-MHC susceptibility locus for multiple sclerosis - the tyrosine kinase 2 (TYK2) gene.

\section{Materials and methods Participants}

Samples independent of those considered in our WTCCC nsSNP GWAS were collected from Australia, Belgium, Norway, the United Kingdom (UK) and the United States of America (USA). A population-specific breakdown together with patient demographics is shown in Table 1. All patients were diagnosed according to the recognised clinical criteria. ${ }^{19,20}$ DNA was extracted using standard methods and all samples were collected with informed written consent and appropriate ethical approval.

\section{Genotyping}

In this analysis, we included the eight SNPs identified in our WTCCC GWAS with a $P$-value $<0.001$ and a further 10 SNPs selected from amongst those with a $P$-value $<0.01$ in the GWAS that were from genes that we considered to be logical candidates for multiple sclerosis. To maximise efficiency and limit unnecessary expenditure, genotyping was performed in two stages. In the first screening stage, we typed all 18 SNPs in 1481 patients, 1879 controls and 806 trio families (from the Belgian, Norwegian and UK1 populations). SNP genotyping was performed using Applied Biosystems TaqMan methodology according to the manufacturer's recommended conditions. A combined analysis of the screening phase data and the original WTCCC data was then completed. In stage 2 of the study, the top five markers identified from this analysis were then followed up in a larger replication set of 2753 patients, 1104 controls and 1247 families from the US, Australian and UK2 (North West UK and South Wales) populations. Power calculations indicate that in both the screening and replication phases, we have greater than $80 \%$ power to find association with a nominal (uncorrected) $P$-value of 0.05 assuming a common risk allele (10\% frequency) with an odds ratio of 1.3 (multiplicative model). ${ }^{21}$ By combining the original WTCCC data with the screening and replication phase data, in total 5213 patients, 4453 unrelated controls and 2053 trio families were considered in the analysis of the top five markers.

\section{Statistical analysis}

The genotyping success rate, Hardy-Weinberg equilibrium and heterozygosity for each marker were established using PEDSTATS. $^{22}$ Mendelian errors in the trio families were identified through PEDCHECK. ${ }^{23}$ In total, five Mendelian errors were identified, one for rs1133400 and four for rs7522061. The case-control data sets were tested for heterogeneity using the Breslow-Day test and a CochranMantel-Haenszel test completed (treating each population specific set of samples as a separate stratum) using StatsDirect statistical software. As no significant heterogeneity was identified between the populations, the raw genotypes were combined and an association analysis was completed using UNPHASED v3.08. ${ }^{24}$

\section{Results}

The 18 SNPs were first tested in the screening phase set of samples. A full list of the SNPs and the results in the screening phase set are shown in Supplementary Table S1. Initial testing showed that the marker rs11080149 (from the $O M G$ gene) deviated significantly from HardyWeinberg equilibrium. As this variant lies in a region of potential copy number variation, the assay was considered to be unreliable and the marker was therefore excluded 
Table 1 Demographic feature of affected individuals

\begin{tabular}{|c|c|c|c|c|c|c|c|c|}
\hline Population & Cases & Controls & Trios & Age (years) & $\begin{array}{c}\text { Average } \\
\text { Disease duration (years) }\end{array}$ & EDSS & $R R / S P \%$ & PP\% \\
\hline \multicolumn{9}{|l|}{ Screening phase } \\
\hline Belgium & 368 & 374 & 113 & 47.0 & 15.0 & 5.4 & 91.2 & 8 \\
\hline Norway & 638 & 1026 & - & 46.3 & 14.3 & 4.8 & 81.4 & 18.6 \\
\hline UK1 & 475 & 479 & 693 & 43.8 & 12.8 & 4.6 & 88.2 & 11.8 \\
\hline WTCCC screen & 979 & 1470 & - & 43.2 & 13.5 & 4.7 & 87.2 & 12.8 \\
\hline \multicolumn{9}{|l|}{ Replication phase } \\
\hline Australia & - & - & 372 & 47.2 & 14.3 & $3.3^{\mathrm{a}}$ & 93.0 & 7 \\
\hline UK2 & 1531 & 561 & - & 44.0 & 13.0 & 4.6 & 91.8 & 8.2 \\
\hline USA & 1222 & 543 & 875 & 44.3 & 12.4 & 4.1 & 94.8 & 5.2 \\
\hline
\end{tabular}

EDSS, expanded disability status scale; RR, relapse remitting; SP, secondary progressive; PP, primary progressive. The case dataset from UK2 includes 880 from the North West of the UK and 516 from South Wales with the remainder coming from across the whole of the UK (with an emphasis on the South east). The control dataset includes 561 individuals from the 1958 birth cohort.

${ }^{a}$ The EDSS was not determined for these patients; instead, the average 'disease steps' measure is shown. ${ }^{34}$

Table 2 Results from association testing of the top five SNPs

\begin{tabular}{|c|c|c|c|c|c|c|c|c|c|c|}
\hline \multirow[b]{2}{*}{ Gene } & \multirow[b]{2}{*}{ Chromosome } & \multirow[b]{2}{*}{ SNP } & \multirow{2}{*}{$\begin{array}{l}\text { Risk } \\
\text { allele }\end{array}$} & \multirow[b]{2}{*}{ Samples } & \multirow{2}{*}{$\begin{array}{l}\text { Screening } \\
\text { P-value }\end{array}$} & \multirow{2}{*}{$\begin{array}{c}\text { Replication }^{\text {b }} \\
\text { P-value }\end{array}$} & \multicolumn{2}{|c|}{ Combined analysis ${ }^{\mathrm{c}}$} & \multirow{2}{*}{$\begin{array}{c}R A F \\
\text { (cases) }\end{array}$} & \multirow{2}{*}{$\begin{array}{c}R A F \\
\text { (controls) }\end{array}$} \\
\hline & & & & & & & P-value & Odds ratio $(95 \% \mathrm{Cl})$ & & \\
\hline \multirow[t]{3}{*}{$I L 7 R$} & 5 & rs6897932 & C & All individuals & $3.5 \times 10^{-4}$ & $4.7 \times 10^{-4}$ & $1.3 \times 10^{-7}$ & $1.42(1.09$ & 0.763 & 0.732 \\
\hline & & & & & & 8.8 & 7.4 & 1.27) & 0.764 & 730 \\
\hline & & & & amilies & 0.5 & 0.1813 & 0.1834 & $1.08(0.96-1.22)$ & 0.761 & 0.747 \\
\hline \multirow[t]{3}{*}{ TYK2 } & 19 & rs34536443 & G & All individuals & $3.9 \times 10^{-4}$ & $2.6 \times 10^{-3}$ & $2.7 \times 10^{-6}$ & $1.32(1.17-1.47)$ & 0.966 & 0.953 \\
\hline & & & & Case-control & $2.1 \times 10^{-3}$ & $3.1 \times 10^{-4}$ & $6.8 \times 10^{-6}$ & $1.39(1.20-1.60)$ & 0.965 & 0.952 \\
\hline & & & & & $4.2 \times 10^{-5}$ & & & $-2.02)$ & 0.970 & 0.954 \\
\hline \multirow[t]{3}{*}{ IFI30 } & 19 & rs11554159 & G & All individuals & $2.0 \times 10^{-3}$ & $8.5 \times 10^{-3}$ & $2.2 \times 10^{-4}$ & $1.10(1.05-1.16)$ & 0.756 & 0.737 \\
\hline & & & & Case-control & $2.1 \times 10^{-3}$ & 0.1208 & 0.017 & $1.08(1.01-1.16)$ & 0.755 & 0.740 \\
\hline & & & & & & $2.5 \times 10^{-4}$ & $10^{-3}$ & $1.17(1.05-1.30)$ & 0.757 & 0.730 \\
\hline \multirow[t]{3}{*}{ FCRL3 } & 1 & rs7522061 & $\mathrm{T}$ & All individuals & $2.4 \times 10^{-4}$ & 0.7557 & $9.1 \times 10^{-3}$ & $1.06(1.01-1.11)$ & 0.545 & 0.528 \\
\hline & & & & Case & $1.0 \times 10^{-3}$ & 0.1432 & $3.4 \times 10^{-4}$ & $1.11(1.05-1.18)$ & 0.549 & 0.523 \\
\hline & & & & Trio families & 0.2350 & 0.1819 & 0.8508 & $0.99(0.90-1.09)$ & 0.536 & 0.541 \\
\hline \multirow[t]{3}{*}{$S L C 4 A 5^{\mathrm{d}}$} & 2 & rs17009792 & C & All individuals & $4.7 \times 10^{-4}$ & 0.7082 & 0.0191 & $1.20(1.03-1.40)$ & 0.980 & 0.974 \\
\hline & & & & Case-control & $9.3 \times 10^{-5}$ & 0.1898 & $1.4 \times 10^{-3}$ & $1.35(1.13-1.63)$ & 0.980 & 0.973 \\
\hline & & & & Trio families & 0.2404 & 0.5411 & 0.7216 & $1.07(0.75-1.52)$ & 0.979 & 0.978 \\
\hline
\end{tabular}

Association analysis was completed using UNPHASED v3.08. The $P$-values presented are uncorrected.

All alleles are specified with respect to the forward strand of the Ensembl genome browser Release 49 (March 2008).

${ }^{a}$ The screening phase includes 1481 patients, 1879 controls and 806 trio families from the Belgian, Norwegian and UK1 populations. The $P$-value is based on the analysis of this new data in combination with the original WTCCC screen data of 979 patients and 1470 controls.

${ }^{\text {b}}$ The replication phase includes 2753 patients, 1104 controls and 1247 families from the US, Australian and UK2 (North West UK and South Wales) populations.

'The combined analysis includes the WTCCC screen data, screening phase and replication phase data.

${ }^{\mathrm{d}}$ Not tested in the Australian population.

from further analysis. All other markers were in HardyWeinberg equilibrium.

An analysis of the original WTCCC data with the screening phase data was completed and the top five SNPs were chosen for further investigation (see Supplementary Table S1). These markers were followed up in the replication data set and a combined analysis of all the available data (screening and replication set samples) was then completed for these five SNPs (Table 2). The population specific data for the top five markers is available in Supplementary Table S2. In this larger analysis, no marker showed deviation from Hardy-Weinberg equilibrium and the average genotyping success rate across these five markers was 99.1\% (range 98.7-99.4\%). There was no statistically significant difference in the genotyping failure rate between cases and controls for any of these markers.

Using the Breslow-Day test, we found no statistically significant evidence for heterogeneity between the different population cohorts or the WTCCC study for any of the top five SNPs. For this reason, it is unsurprising that the results from a Cochran-Mantel-Haenszel analysis showed 
no meaningful difference from the results obtained, when treating all the data as if they had been collected from a single population.

The most significant association that we observed was with rs6897932 from the $I L 7 R$ gene $\left(P=1.3 \times 10^{-7}\right)$. As this marker is already confirmed as a susceptibility locus ${ }^{11,12}$ in multiple sclerosis it provides a useful positive control for our study and illustrates that screens based on nsSNPs are capable of identifying genuine associations.

The next most strongly associated marker was rs34536443 from exon 21 of the TYK 2 gene $\left(P=2.7 \times 10^{-6}\right)$. As with the $I L 2 R$ and $I L 7 R$, the common allele increases risk at rs34536443. This risk allele has a frequency of $95.3 \%$ in the background population and increases risk with an odds ratio of $1.32(1.17-1.47)$. In a stratified analysis of the individual replication cohorts, the trend for association was in the same direction for all cohorts, with the only exception being the Belgian population (the smallest cohort analysed).

In the final analysis of all available data, evidence for association was either substantially reduced or largely uninfluenced for each of the other markers studied. One marker retained modest evidence for association (rs11554159 from IFI30, $P=2.2 \times 10^{-4}$ ) and may represent a genuine association of smaller effect, but very large sample sizes will be needed to confirm such an association.

\section{Discussion}

This study reports the first attempt to explore the results that emerged from our WTCCC nsSNP GWAS. Through the typing of selected markers in a large independent cohort of cases, controls and trio families, we have shown evidence of a novel association with rs34536443 in the TYK 2 gene.

Tyrosine kinase 2, part of the Janus family of tyrosine kinases, has a crucial role in signal transduction for a wide range of cytokines, including type 1 interferons, IL10 and IL12. ${ }^{25,26}$ TYK2 deficiency in humans results in severe combined immunodeficiency (SCID), ${ }^{27}$ with reduced Th1 (and possibly Th17 immune responses) and increased production of Th2 cytokines. ${ }^{25}$ TYK 2 variants have been associated with susceptibility to the autoimmune disease, systemic lupus erythematosus. ${ }^{28,29}$ The detrimental autoimmune response generated by TYK2 is further shown in Tyk2-deficient mice that are shown to be resistant to autoimmune arthritis. ${ }^{30}$ The SNP rs34536443 is located in exon 21 and codes either a conserved proline (major allele) or alanine at position 1104, in the tyrosine kinase domain. $1104 \mathrm{~A}$ is predicted to be 'probably damaging' based on the program Polymorphism Phenotyping (PolyPhen) and 'intolerant' based on Sorting Intolerant from Tolerant (SIFT). The shift to a Th1 pro-inflammatory immune response in multiple sclerosis is believed to lead to cell destruction and consequent pathology. The reduced TYK2 function of the $1104 \mathrm{~A}$ variant could lead to a shift to a protective Th2 response, and/or reduced Th1 or Th17 activation. As the minor allele frequency is higher in the control population, this further supports the possibility that the rs34536443 minor allele may be protective.

IFI30 or IFN $\gamma$-inducible lysosomal thiol reductase (GILT) is a prime candidate gene in multiple sclerosis as it has a vital role in antigen processing through MHC class II and upregulated expression on macrophages and microglia in active demyelinating lesions in multiple sclerosis. ${ }^{31}$ GILT is expressed constitutively in antigen presenting cells and is induced by inflammatory cytokines, such as IFN $\gamma, \mathrm{TNF} \alpha$ and IL1 $\beta$ in other cell types. ${ }^{32}$ Studies in mice have shown that GILT deficient $\mathrm{T}$ cells show stronger T-cell activation and increased proliferation, suggesting a role for GILT in modifying the immune response by controlling T-cell activation. ${ }^{33}$ Although the strength of association for rs11554159 was reduced compared with the initial nsSNP screen (despite the trend for association being in the same direction for all cohorts analysed), modest evidence for association remained. As initial findings tend to overinflate the effect size, our replication cohort may be too limited in power to confirm this association. However, given these findings combined with the involvement of IFI30 in the immune response, this gene warrants further analysis in a much larger cohort.

In summary, we have identified a novel association with the TYK2 gene. The power to detect the small genetic effect attributed to this SNP has been crucially dependent on the large sample sizes analysed in this study. The consequence of the rs34536443 SNP on TYK2 function and expression in patients with multiple sclerosis needs to be explored, together with the role this gene may have in autoimmunity in general.

\section{Acknowledgements}

We thank members of the Association of British Neurologists for notifying families. We acknowledge contributions from the International Multiple Sclerosis Genetics Consortium. This work was supported by the Medical Research Council (G0700061) and the National Institute of Health (NS 049477-01A1). We acknowledge use of DNA from the British 1958 Birth Cohort collection, funded by the Medical Research Council grant G0000934 and the Wellcome Trust grant 068545/Z/02. The Norwegian Bone Marrow Donor Registry is acknowledged for collaboration in the establishment of the Norwegian control material. AG is a Postdoctoral Fellow, EB a Research Assistant, and BD a Clinical Investigator of the Research Foundation Flanders (FWO-Vlaanderen). ARL and HFH are supported by The Research Council of Norway (166005/V5), the Ulleval University Hospital Scientific Advisory Council and by the Odd Fellow MS society. This study makes use of data generated by The Wellcome Trust CaseControl Consortium. A full list of the investigators who contributed to the generation of the data is available from www.wtccc.org.uk. Funding for the project was provided by The Wellcome Trust under award 076113 and reported in Nature 2007; 447; 661-78.

\section{Websites}

StatsDirect statistical software - http://www.statsdirect.com. PolyPhen - http://genetics.bwh.harvard.edu/pph/. SIFT - http://blocks.fhcrc.org/sift/SIFT.html. 


\section{References}

1 Compston A, Coles A: Multiple sclerosis. Lancet 2008; 372: $1502-1517$.

2 Hogancamp WE, Rodriguez M, Weinshenker BG: The epidemiology of multiple sclerosis. Mayo Clin Proc 1997; 72: 871-878.

3 Ebers GC, Sadovnick AD, Risch NJ: A genetic basis for familial aggregation in multiple sclerosis. Canadian Collaborative Study Group. Nature 1995; 377: 150-151.

4 Ebers GC, Yee IM, Sadovnick AD, Duquette P: Conjugal multiple sclerosis: population-based prevalence and recurrence risks in offspring. Canadian Collaborative Study Group. Ann Neurol 2000; 48: 927-931.

5 Sadovnick AD, Ebers GC, Dyment DA, Risch NJ: Evidence for genetic basis of multiple sclerosis. The Canadian Collaborative Study Group. Lancet 1996; 347: 1728-1730.

6 Jersild C, Svejgaard A, Fog T: HL-A antigens and multiple sclerosis. Lancet 1972; 1: 1240-1241.

7 Compston A, Confavreux C, Lassmann $\mathrm{H}$ et al: McAlpine's Multiple Sclerosis. Churchill Livingstone: Edinburgh, 2005.

8 Sawcer S, Ban M, Maranian M et al: A high-density screen for linkage in multiple sclerosis. Am J Hum Genet 2005; 77: $454-467$.

9 Yang Q, Khoury MJ, Friedman J, Little J, Flanders WD: How many genes underlie the occurrence of common complex diseases in the population? Int J Epidemiol 2005; 34: 1129-1137.

10 Lindsey JW: Familial recurrence rates and genetic models of multiple sclerosis. Am J Med Genet A 2005; 135: 53-58.

11 Gregory SG, Schmidt S, Seth P et al: Interleukin 7 receptor alpha chain (IL7R) shows allelic and functional association with multiple sclerosis. Nat Genet 2007; 39: 1083-1091.

12 Lundmark F, Duvefelt K, Iacobaeus E et al: Variation in interleukin 7 receptor alpha chain (IL7R) influences risk of multiple sclerosis. Nat Genet 2007; 39: 1108-1113.

13 International Multiple Sclerosis Genetics Consortium T: Risk alleles for multiple sclerosis identified by a genome wide study. N Engl J Med 2007; 357: 851-862.

14 The International Multiple Sclerosis Genetics Consortium: Refining genetic associations in multiple sclerosis. Lancet Neurol 2008; 7: $567-569$.

15 Hafler JP, Maier LM, Cooper JD et al: CD226 Gly307Ser association with multiple autoimmune diseases. Genes Immun 2009; 10: 5-10.

16 Rubio JP, Stankovich J, Field J et al: Replication of KIAA0350, IL2RA, RPL5 and CD58 as multiple sclerosis susceptibility genes in Australians. Genes Immun 2008; 9: 624-630.

17 The International Multiple Sclerosis Genetics Consortium: The expanding genetic overlap between multiple sclerosis and type I diabetes. Genes Immun 2009; 10: 11-14.

18 Burton PR, Clayton DG, Cardon LR et al: Association scan of 14,500 non synonymous SNPs in four diseases identifies autoimmunity variants. Nat Genet 2007; 39: 1329-1337.
19 Poser CM, Paty DW, Scheinberg L et al: New diagnostic criteria for multiple sclerosis: guidelines for research protocols. Ann Neurol 1983; 13: 227-231.

20 McDonald WI, Compston A, Edan G et al: Recommended diagnostic criteria for multiple sclerosis: guidelines from the International Panel on the diagnosis of multiple sclerosis. Ann Neurol 2001; 50: 121-127.

21 Purcell S, Cherny SS, Sham PC: Genetic Power Calculator: design of linkage and association genetic mapping studies of complex traits. Bioinformatics 2003; 19: 149-150.

22 Wigginton JE, Abecasis GR: PEDSTATS: descriptive statistics, graphics and quality assessment for gene mapping data. Bioinformatics 2005; 21: 3445-3447.

23 O'Connell JR, Weeks DE: PedCheck: a program for identification of genotype incompatibilities in linkage analysis. Am J Hum Genet 1998; 63: 259-266.

24 Dudbridge F: Pedigree disequilibrium tests for multilocus haplotypes. Genet Epidemiol 2003; 25: 115-121.

25 Minegishi Y, Saito M, Morio T et al: Human tyrosine kinase 2 deficiency reveals its requisite roles in multiple cytokine signals involved in innate and acquired immunity. Immunity 2006; 25: $745-755$.

26 Tokumasa N, Suto A, Kagami S et al: Expression of Tyk2 in dendritic cells is required for IL-12, IL-23, and IFN-gamma production and the induction of Th1 cell differentiation. Blood 2007; 110: 553-560.

27 Watford WT, O'Shea JJ: Human tyk2 kinase deficiency: another primary immunodeficiency syndrome. Immunity 2006; 25: 695-697.

28 Graham DS, Akil M, Vyse TJ: Association of polymorphisms across the tyrosine kinase gene, TYK2 in UK SLE families. Rheumatology (Oxford) 2007; 46: 927-930.

29 Sigurdsson S, Nordmark G, Goring HH et al: Polymorphisms in the tyrosine kinase 2 and interferon regulatory factor 5 genes are associated with systemic lupus erythematosus. Am J Hum Genet 2005; 76: 528-537.

30 Shaw $\mathrm{MH}$, Boyartchuk V, Wong S et al: A natural mutation in the Tyk2 pseudokinase domain underlies altered susceptibility of B10.Q/J mice to infection and autoimmunity. Proc Natl Acad Sci USA 2003; 100: 11594-11599.

31 Satoh JI, Obayashi S, Misawa Tet al: Neuromyelitis optica/Devic's disease: gene expression profiling of brain lesions. Neuropathology 2008; 28: 561-576.

32 Phan UT, Arunachalam B, Cresswell P: Gamma-interferoninducible lysosomal thiol reductase (GILT). Maturation, activity, and mechanism of action. J Biol Chem 2000; 275: 25907-25914.

33 Barjaktarevic I, Rahman A, Radoja S et al: Inhibitory role of IFNgamma-inducible lysosomal thiol reductase in T cell activation. Immunol 2006; 177: 4369-4375.

34 Hohol MJ, Orav EJ, Weiner HL: Disease steps in multiple sclerosis: a longitudinal study comparing disease steps and EDSS to evaluate disease progression. Mult Scler 1999; 5: 349-354.

Supplementary information accompanies the paper on European Journal of Human Genetics website (http://www.nature.com/ejhg) 